\title{
Diverse clinical characteristics of Aspergillus growth in patients with cystic fibrosis
}

\author{
Nagehan Emiralioğlu' ${ }^{1 \oplus}$, Deniz Doğru${ }^{1 \oplus}$, Özlem Doğan ${ }^{2 \odot}$, Dolunay Gülmez ${ }^{2 \odot, ~}$

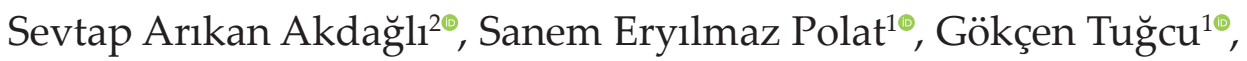 \\ Mina Gharibzadeh $\mathrm{H}_{\text {ızal }}{ }^{1 \odot}$, Ebru Yalçın ${ }^{1 \odot}$, Uğur Özçelik ${ }^{1 \odot}$, Burçin Şener ${ }^{2 \odot}$, \\ Nural Kiper ${ }^{1 \odot}$ \\ Departments of ${ }^{1}$ Pediatric Pulmonology and ${ }^{2}$ Clinical Microbiology, Hacettepe University Faculty of Medicine, Ankara, Turkey.
}

\begin{abstract}
Background and objectives. Patients with cystic fibrosis (CF) have a varying spectrum of clinically significant Aspergillus disease in addition to allergic bronchopulmonary aspergillosis (ABPA). Here we aimed to review the different clinical phenotypes related with Aspergillus growth on the airway culture of patients with $\mathrm{CF}$, we also aimed to investigate the effect of Aspergillus growth on lung function tests.
\end{abstract}

Method. The medical records of 100 patients with CF who had Aspergillus growth on airway culture within the period of April 2001 and June 2016 were retrospectively analyzed. Age, gender, symptoms, physical examination findings, pulmonary function tests, the diagnosis of ABPA, and airway culture results were recorded for every visit. Patients with Aspergillus growth on airway cultures were classified into different groups as ABPA, Aspergillus sensitization, Aspergillus colonization and Aspergillus bronchitis.

Results. Medical records of 83 patients and 147 sputum cultures were attained from 100 patients. The mean age of the patients was 17.6 \pm 7.6 years and the mean age of the first Aspergillus growth in sputum culture was $12.5 \pm 6.7$ years. At first isolation, Aspergillus fumigatus SC was the most common Aspergillus SC in sputum (76.3\%) and $14.5 \%$ of these patients required hospitalization. Aspergillus sensitization was diagnosed in $3.6 \%(n=3)$ of the patients. Aspergillus colonization was diagnosed in $18.1 \%(\mathrm{n}=15)$ of all patients and led to a decline in FEV1\%, FVC $\%$ and FEF25-75\% which was not statistically significant, furthermore. ABPA was detected in 9.6\% ( $\mathrm{n}=8)$ of all patients and led to a statistically significant decline in FEV1\% ( $p=0.02)$; nonsignificant decline in FVC $\%$ and FEF25-75\%. Aspergillus bronchitis was detected in 43.4\% $(n=36)$ of all patients and led to nonsignificant decline in $\mathrm{FEV} 1 \%$, FVC\% and FEF25-75\%.

Conclusion. ABPA is recognized as the most common Aspergillus associated disorder in CF patients and is related to deteriorated pulmonary function tests; however Aspergillus colonization and bronchitis may also be associated with worsening lung function.

Key words: cystic fibrosis, aspergillus colonization, aspergillus sensitization, ABPA, aspergillus bronchitis.

Cystic fibrosis (CF) is a systemic chronic disease and abnormalities in CFTR (Cystic fibrosis transmembraneconductanceregulator)function affect interactions between the epithelial surface and microorganisms such as fungi. ${ }^{1}$ Conidial

\footnotetext{
$凶$ Nagehan Emiralioğlu

drnagehan@yahoo.com
}

Received 17th October 2019, revised 24th December 2019, accepted 25th December 2019. spores are usually cleared by airway epithelium; however, in CF due to disruption of epithelial barrier and germination of the fungi induce inflammatory response. Increased mucus viscosity and lung injury result in an ongoing cycle of infection, inflammation and pulmonary damage. ${ }^{1,2}$ Aspergillus, the most commonly detected filamentous fungi in CF induces this abnormal pulmonary inflammation. ${ }^{2}$ The main Aspergillus- associated clinical manifestations 
in $\mathrm{CF}$ are Allergic Bronchopulmonary Aspergillosis (ABPA), Aspergillus sensitization, Aspergillus colonization, Aspergillus bronchitis and, rarely aspergilloma and invasive disease. ${ }^{1}$

ABPA is a hypersensitivity response to Aspergillus antigens; the prevalence of ABPA changes from 2 to $8 \%$, and lung function has been shown to deteriorate over time related with ABPA. The increasing prevalence of Aspergillus sensitization has also been reported from 20 to $65 \%$ recently and separate from CFABPA, Aspergillus sensitization has significant clinical effects on lung function and patient morbidity. ${ }^{1,3,4}$ The term 'colonization' defines the presence of a microorganism which causes neither symptoms nor immunologic response. Aspergillus bronchitis was first described by Shoseyov et al. ${ }^{5}$ for patients with CF who had respiratory exacerbations non-responsive to appropriate antimicrobial therapy, cultured Aspergillus from sputum and responded to antifungal therapy. Few studies have addressed the role of Aspergillus on lung function in CF. However, the effect of colonization and Aspergillus bronchitis on lung function other than ABPA is not clear. ${ }^{1}$

Recommendations for the treatment of ABPA in patients with $\mathrm{CF}$ are now clearly described; however, the pathogenic role of Aspergillus and the benefit of treatment of an Aspergillus bronchial infection remain to be clarified in clinical conditions other than ABPA. There is also no consensus on the antifungal therapies of CF patients presenting with persistent Aspergillus-positive cultures or those with sensitization to Aspergillus. ${ }^{6-8}$

Here we aimed to review the different clinical phenotypes related with Aspergillus growth on the airway culture of patients with $\mathrm{CF}$ and we also aimed to evaluate the effect of Aspergillus growth on lung function of these patients. Our second aim was to review the risk factors affecting the growth of Aspergillus in airway culture of patients with CF.

\section{Material and Methods}

\section{Subjects}

In this retrospective cohort study, medical records of children with a diagnosis of $\mathrm{CF}$ ( two positive sweat chloride tests $(\geq 60 \mathrm{mmol} / \mathrm{L})$ and/ or genotype confirmation) treated at our tertiary care centre between April 2001 and June 2016 who had Aspergillus growth in sputum culture were evaluated.

Age, gender, symptoms, physical examination findings, pulmonary function tests including Forced expiratory volume in the first second (FEV1), Forced vital capacity (FVC), Forced expiratory flow between $25 \%$ and $75 \%$ of the FVC (FEF25-75), and airway culture results were recorded for every visit. "Increase in symptoms" were defined as increased cough, sputum, dyspnea or fever; new physical examination findings were also defined newly detected lung sounds like rale or rhonchi that were not defined on clinical follow-up of the patient. Immunological data included total immunglobulin E (IgE), specific anti - $A$. fumigatus IgE in all patients and specific anti - A. fumigatus IgG only in two patients as it is not routinely performed in our hospital. Skin prick test reactivity to Aspergillus antigen and treatment modalities were also recorded in all patients. Patients were followed every three months. Spirometric data were recorded for three years.

\section{Microbiological methods}

Airway cultures were taken at each visit to our centre from all patients. Respiratory cultures of the patients were inoculated onto 5\% sheep blood agar, MacConkey agar, chocolate agar with bacitracin, mannitol salt agar and Burkholderia cepacia selective agar. Bacterial isolates were identified by conventional methods and automated bacterial identification systems (BD Phoenix, USA; or VITEK 2, bioMerieux, France). Mycological cultures were plated on Sabouraud dextrose agar (SDA) and incubated at $35 \pm 2^{\circ} \mathrm{C}$ and $25 \pm 2^{\circ} \mathrm{C}$ for seven 
days. Identification of Aspergillus strains at species complex (SC) level were performed using conventional mycological methods. ${ }^{9}$

\section{Definitions}

Patients with Aspergillus growth on airway cultures were classified into different groups according to criteria defined by Baxter et al. and Shoseyov et al. ${ }^{5,10,11}$ As Aspergillus specific IgG testing is not available in our hospital, we could not use Aspergillus specific IgG for this classification.

1- Aspergillus colonization: Two or more Aspergillus fumigatus SC positive cultures in any 12 months during the study period without elevation of total IgE and A. fumigatus specific IgE.

2- Aspergillus sensitization: Elevation of total IgE and $A$. fumigatus specific IgE without fulfilling the diagnostic criteria of ABPA. A. fumigatus specific IgG is not elevated. Because of missing data of Aspergillus specific IgG in our cohort, we defined sensitization depending on clinical criteria, total IgE and A. fumigatus specific IgE.

3- Aspergillus bronchitis: Due to missing examinations of sputum galactomannan, Aspergillus specific DNA and serum specific IgG in our cohort, Aspergillus bronchitis was defined according to these criteria; clinical deterioration with positive sputum cultures for Aspergillus, no antibiotic treatment response, total serum IgE level $<200 \mathrm{kU} / \mathrm{l}$, no observation of new pulmonary infiltrates and appropriate antifungal treatment response with the exclusion of ABPA. ${ }^{5,12}$ Because of missing data of Aspergillus specific IgG; differential diagnosis with colonization was made depending on clinical deterioration of these patients.

4- ABPA diagnosis was based on the criteria published in the ABPA consensus paper that included five or more of the following: (a) Acute or subacute clinical deterioration not attributable to another etiology; (b) Total serum IgE concentration higher than $500 \mathrm{IU} /$ $\mathrm{ml}$; (c) Skin prick test reactivity to Aspergillus; (d) Presence of serum IgE antibodies to $A$. fumigatus (higher than $0.35 \mathrm{kU} / \mathrm{l}$ ); (e) Precipitins or IgG antibodies to A. fumigatus; (f) New or recent pulmonary infiltrates, mucus plugging, or bronchiectasis with no response to antibiotics and physiotherapy. ${ }^{13}$

This study was approved by the local institutional review board in 2018 with the number GO/18/271-43.

\section{Statistical analysis}

Statistical analyses were performed using the Statistical Package for the Social Sciences (SPSS) version 23.0 (SPSS Inc., Chicago, IL, USA). The Kolmogorov-Smirnov test was used to determine whether the variable had a normal distribution. Means and standard deviations (SD) were calculated for continuous and numbers (percentages) for categorical data. More than two independent groups were compared using One-Way Anova and Tukey post-hoc test for normal continuous variables or Kruskal-Wallis test and Bonferroni adjusted Mann-Whitney $U$ test for non-normal continuous variables. Mann-Whitney $U$ test was used to compare continuous variables between independent two groups. Chi squared test was performed to compare proportions between groups. Logistic regression was used to analyse the effects of potential factors on Aspergillus growth. Repeated measures of variance analyses were used for yearly lung function test changes of patients. Mean FEV1 and FVC decline were expressed as the annual average change in $\mathrm{FEV} 1 \%$ and $\mathrm{FVC} \%$ during one year period by simple regression analysis. Statistical tests were two-sided and statistical significance was accepted at $\mathrm{p}<0.05$.

\section{Results}

There were 100 patients with Aspergillus growth in airway culture during the study period. From these, medical records of 83 patients and 147 airway cultures of these patients were attained. The mean age of all patients was $17.6 \pm 7.7$ years and the mean age of the first Aspergillus growth 
in airway culture was $12.5 \pm 6.7$ years. Male to female ratio was $47 / 36.26 .5 \%(n=22)$ of patients have F508 homozygous deletion, 16.8\% ( $\mathrm{n}=14)$ of patients have F508 heterozygous deletion and $56.4 \%(n=47)$ of patients have other CFTR mutations.

Fifty-three patients had Aspergillus growth more than once. Bronchiectasis was detected in $91.6 \%(n=76)$ and pancreatic insufficiency was noticed in $100 \% \quad(n=83)$ of the patients. Chronic Pseudomonas aeruginosa and chronic Staphylococcus aureus colonization were detected in $49.4 \%(n=41)$ and $48.2 \%(n=40)$ of patients, respectively.

At first isolation, A. fumigatus SC was the most common SC of Aspergillus in airway cultures (76.3\%). $25.3 \%(n=21)$ of the patients had Aspergillus growth once a time and asymptomatic at first isolation. The remaining 62 patients were classified into different groups. ABPA was diagnosed in $9.6 \%$ of patients $(n=8)$ and totally ABPA was diagnosed 24 times of these eight patients in this period. Aspergillus sensitization was detected in three patients and Aspergillus colonization was detected in 18.1\% $(\mathrm{n}=15)$ of the patients. Aspergillus bronchitis was detected in $43.4 \%(n=36)$ of the patients. Table I shows the clinical characteristics of patients at first isolation according to diagnosis of ABPA, Aspergillus colonization, Aspergillus bronchitis

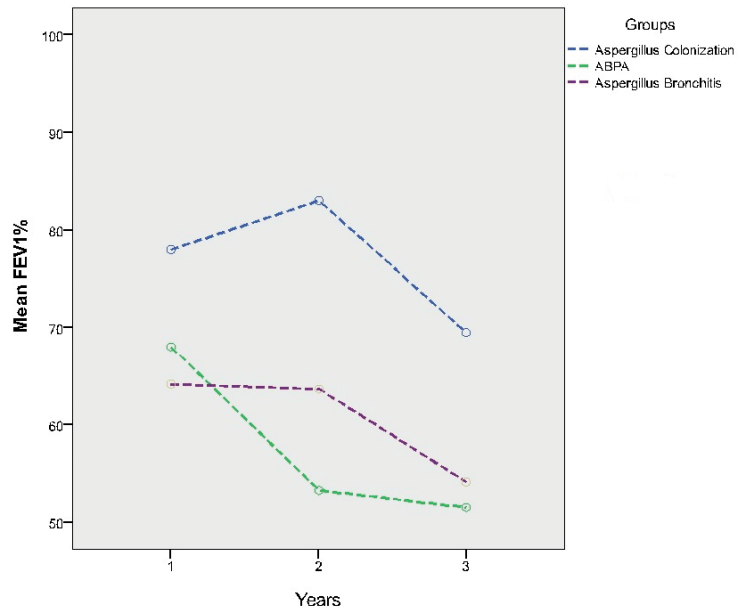

Fig. 1. Change of FEV1\% in patients with ABPA, Aspergillus colonization and Aspergillus bronchitis. and Aspergillus sensitization.

In three years of follow up, ABPA led to statistically significant decline in FEV1\% ( $p=$ 0.02). Although decline in $\mathrm{FVC} \%$ and FEF25$75 \%$ were also present; these findings were not statistically significant.

Aspergillus colonization and Aspergillus bronchitis also led to decline in FEV1\%, FVC\% and FEF25-75\% which was not statistically significant in these patients furthermore. Table II shows the change of lung function tests within the time in patients with ABPA, Aspergillus colonization and Aspergillus bronchitis. Figure 1 and Figure 2 also show the graphic of the lung function tests within the groups of ABPA, Aspergillus colonization and Aspergillus bronchitis. The decline of FEV1\% ( $\mathrm{p}=$ $0.45)$ and $\mathrm{FVC} \%(\mathrm{p}=0.10)$ were not statistically significant between the groups furthermore. We did not include the change of lung functions in the group with Aspergillus sensitization due to small number of patients in this group. The mean FEV1 decline within the groups of ABPA, Aspergillus bronchitis and Aspergillus colonization was $5.5 \%, 3.3 \%, 2.2 \%$ per year and the mean FVC decline within the groups of ABPA, Aspergillus bronchitis and Aspergillus colonization was $6.2 \%, 3.8 \%, 0.3 \%$ per year respectively in the following three years.

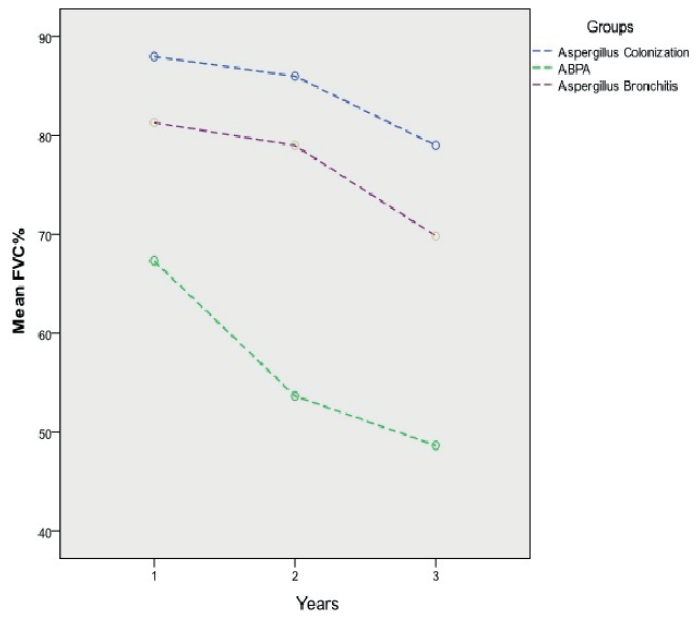

Fig. 2. Change of $\mathrm{FVC} \%$ in patients with $\mathrm{ABPA}$, Aspergillus colonization and Aspergillus bronchitis. 
Table I. Demographic and clinical characteristics of patients with ABPA, Aspergillus colonization, Aspergillus bronchitis and Aspergillus sensitization.

\begin{tabular}{|c|c|c|c|c|c|}
\hline & ABPA & $\begin{array}{l}\text { Aspergilllus } \\
\text { colonization }\end{array}$ & $\begin{array}{l}\text { Aspergillus } \\
\text { bronchitis }\end{array}$ & $\begin{array}{l}\text { Aspergillus } \\
\text { sensitization }\end{array}$ & $\mathrm{p}$ \\
\hline Number of patients, $n$ & 8 & 15 & 36 & 3 & \\
\hline Age at last visit (years), & $18.6( \pm 4.2)$ & $18.6( \pm 4.5)$ & $18.4( \pm 5.5)$ & $19.7( \pm 5)$ & 0.95 \\
\hline Mean $( \pm$ SD) (Min-Max) & $(13.5-24)$ & $(7.5-27)$ & $(6.5-29)$ & $(14.5-24.5)$ & 0.90 \\
\hline Age at first Aspergillus isolation (years), & $13.6( \pm 4.1)$ & $12.3( \pm 6.9)$ & $13.6( \pm 5)$ & $11.2( \pm 5.1)$ & 0.71 \\
\hline Mean $( \pm$ SD) $($ Min-Max $)$ & $(8-21)$ & $(3-27)$ & $(5-27)$ & $(7.5-17)$ & \\
\hline CFTR mutation, $\mathrm{n}$ & & & & & 0.66 \\
\hline F508 del homozygous & 2 & 3 & 10 & 2 & \\
\hline F508 del heterozygous & 1 & 5 & 4 & 1 & \\
\hline Other & 4 & 7 & 22 & 0 & \\
\hline Increase in symptoms at first isolation, $\mathrm{n}(\%)$ & $7(87.5 \%)$ & $6(40 \%)$ & $23(63.9 \%)$ & 0 & 0.01 \\
\hline $\begin{array}{l}\text { New physical examination finding } \\
\text { at first isolation, } \mathrm{n}(\%)\end{array}$ & $5(62.5 \%)$ & $5(33.3 \%)$ & $20(55.6 \%)$ & 0 & 0.10 \\
\hline Bronchiectasis, n (\%) & $8(100 \%)$ & $15(100 \%)$ & $35(97.2 \%)$ & $3(100 \%)$ & 0.70 \\
\hline Chronic $P$. aeruginosa colonization, $\mathrm{n}(\%)$ & $5(62.5 \%)$ & $9(60 \%)$ & $17(47.2 \%)$ & $3(100 \%)$ & 0.23 \\
\hline Chronic S. aureus colonization, $\mathrm{n}(\%)$ & $4(50 \%)$ & $8(53.3 \%)$ & $15(41.7 \%)$ & $1(33.3 \%)$ & 0.77 \\
\hline \multicolumn{6}{|l|}{$\begin{array}{l}\text { Pulmonary function tests at first isolation, } \\
\text { Mean }( \pm S D)\end{array}$} \\
\hline FEV1\% & $55( \pm 24)$ & $76( \pm 10)$ & $60( \pm 19)$ & $64( \pm 45)$ & 0.22 \\
\hline $\mathrm{FVC} \%$ & $59( \pm 22)$ & $87( \pm 11)$ & $68( \pm 20)$ & $64( \pm 10)$ & 0.08 \\
\hline FEF25-75\% & $47( \pm 24)$ & $62( \pm 25)$ & $49( \pm 23)$ & $45( \pm 13)$ & 0.68 \\
\hline Aspergillus SC, $\mathrm{n}$ & & & & & 0.004 \\
\hline A. fumigatus & 2 & 13 & 27 & 1 & \\
\hline A. flavus & 4 & 0 & 3 & 2 & \\
\hline A. terreus & 1 & 0 & 3 & 0 & \\
\hline A. niger & 0 & 0 & 2 & 0 & \\
\hline Aspergillus spp. & 1 & 0 & 1 & 0 & \\
\hline $\begin{array}{l}\text { Coinfection with other pathogens at first } \\
\text { isolation, } \mathrm{n}\end{array}$ & & & & & 0.59 \\
\hline No growth & 1 & 2 & 3 & 0 & \\
\hline S. aureus & 2 & 4 & 12 & 0 & \\
\hline H. influenza & 0 & 0 & 1 & 0 & \\
\hline S. pneumonia & 0 & 1 & 0 & 0 & \\
\hline P. aeruginosa & 3 & 4 & 8 & 3 & \\
\hline Acinetobacter & 0 & 0 & 0 & 0 & \\
\hline S. aureus and $P$. aeruginosa coinfection & 2 & 4 & 12 & 0 & \\
\hline Inhaled antibiotic at first isolation, $\mathrm{n}$ & & & & & 0.90 \\
\hline Inhaled tobramycin & 4 & 5 & 11 & 1 & \\
\hline Inhaled colimycin & 0 & 1 & 1 & 0 & \\
\hline Treatment at first isolation, $\mathrm{n}$ & & & & & 0.001 \\
\hline Oral antifungal treatment & 6 & 12 & 32 & 3 & \\
\hline Intravenous antifungal treatment & 2 & 0 & 0 & 0 & \\
\hline Steroid treatment & 8 & 0 & 0 & 0 & \\
\hline Hospitalization, $\mathrm{n}$ & 3 & 2 & 7 & 0 & 0.42 \\
\hline
\end{tabular}


Table II. Lung functions of patients in different groups.

\begin{tabular}{|c|c|c|c|c|c|c|}
\hline Lung function test & $\begin{array}{l}\text { Aspergillus } \\
\text { bronchitis } \\
(\mathrm{n}=12)\end{array}$ & $\mathrm{p}$ & $\begin{array}{l}\text { Aspergillus } \\
\text { colonization } \\
(\mathrm{n}=6)\end{array}$ & $\mathrm{p}$ & $\begin{array}{l}\text { ABPA } \\
(n=5)\end{array}$ & $\mathrm{p}$ \\
\hline$\overline{\mathrm{FEV}} \%$, mean $\pm \mathrm{SD}$ & & 0.41 & & 0.09 & & 0.02 \\
\hline First year & $64.1 \pm 18.4$ & & $83 \pm 10$ & & $68 \pm 17$ & \\
\hline Second year & $63.6 \pm 19.4$ & & $73.6 \pm 8$ & & $53.2 \pm 12.5$ & \\
\hline Third year & $54.1 \pm 23.5$ & & $76.3 \pm 7.7$ & & $51.5 \pm 14.6$ & \\
\hline $\mathrm{FVC} \%$, mean $\pm \mathrm{SD}$ & & 0.31 & & 0.14 & & 0.22 \\
\hline First year & $81.3 \pm 11.9$ & & $85.6 \pm 0.5$ & & $67.3 \pm 23.5$ & \\
\hline Second year & $79 \pm 9.9$ & & $76 \pm 7.2$ & & $53.6 \pm 13.2$ & \\
\hline Third year & $69.8 \pm 21.5$ & & $84.6 \pm 5$ & & $48.6 \pm 17.7$ & \\
\hline FEF $25-75 \%$, mean $\pm S D$ & & 0.41 & & 0.26 & & 0.12 \\
\hline First year & $49.8 \pm 22.3$ & & $62.3 \pm 10.5$ & & $56 \pm 17$ & \\
\hline Second year & $42 \pm 18.8$ & & $47.3 \pm 5.5$ & & $35 \pm 7.7$ & \\
\hline Third year & $39.8 \pm 20.1$ & & $58.3 \pm 10.6$ & & $40 \pm 13.7$ & \\
\hline
\end{tabular}

ABPA: allergic bronchopulmonary aspergillosis, FEV1: forced expiratory volume in the first second,

FVC: forced vital capacity, FEF25-75: forced expiratory flow between $25 \%$ and $75 \%$ of the FVC.

The risk of Aspergillus growth in airway cultures was not affected significantly by the age, CFTR mutation, symptoms, physical examination findings, radiological findings, sputum culture results and inhaled antibiotic treatment in logistic regression analysis.

\section{Discussion}

A. fumigatus SC is one of the main fungal subgroup found in CF airways and results in different clinical states and wide range of host responses affecting the progression of CF lung disease. In this retrospective cohort, we reviewed different clinical phenotypes of Aspergillus growth on the airway culture of patients with CF classified Aspergillus colonization, Aspergillus bronchitis, Aspergillus sensitization and ABPA.,11

The prevalence of Aspergillus colonization ranges from 10 to $57 \%$ with increasing age in CF patients. ${ }^{14}$ de Vrankrijker et al. ${ }^{15}$ reported a cohort of $259 \mathrm{CF}$ patients and found 61 (23.5\%) of these patients had $A$. fumigatus colonization according to the criteria of having more than $50 \%$ positive sputum cultures in a given year. The prevalence of Aspergillus colonization in pediatric CF patients is not well established yet. In our cohort we reported $18.1 \%$ of patients with Aspergillus colonization. Saunders et al. ${ }^{16}$ also reported $22 \%$ of children fulfilled the criteria for A. fumigatus colonization within eight years of study period. The prevalence of ABPA in patients with CF varied from 3 to $25 \%$, and the prevalence of Aspergillus sensitization varied from 20 to $65 \%$ in a metaanalysis by Maturu et al. $^{17}$ Our cohort revealed $9.6 \%$ of ABPA patients. In our cohort we had three patients with sensitization. Baxter et al. ${ }^{10}$ and Shoseyov et al. ${ }^{5}$ defined Aspergillus bronchitis as repeatedly Aspergillus growth in sputum samples without hypersensitivity to Aspergillus and with persistent respiratory symptoms and no response to antibiotics in patients with CF. Baxter et al. $^{10}$ reported $30 \%$ of patients with Aspergillus bronchitis in their adult cohort. In our cohort we also reported $43.4 \%$ of patients with Aspergillus bronchitis similar to these results.

Few studies have reported the association between age and risk of Aspergillus isolation. Saunders et al. ${ }^{16}$ reported that the isolation of A. fumigatus was common in children older than 10 years and most of the older children 
eventually became persistently colonized. We also found that the mean age of the first Aspergillus growth in airway culture was 12 \pm 6.6 years similar to this study. In our cohort the risk of Aspergillus colonization was not affected significantly by age, CFTR mutation, symptoms, physical examination findings, radiological findings, sputum culture results and inhaled antibiotic treatment. Colonization of the airways by A. fumigatus usually develops after chronic colonization with $P$. aeruginosa. Noni et al. ${ }^{18}$ concluded that their patients with colonization were not found to have significantly higher $P$. aeruginosa colonization and inhaled antibiotic treatment rates similar to our study. However Bargon et al. ${ }^{19}$ suggested that prophylactic antibiotics may cause $A$. fumigatus colonization. Another study by Burns et al. $^{20}$ concluded the use of inhaled tobramycin increased A. fumigatus isolation in the treatment group, at the end of the study period. According to our findings, it is difficult to say whether there is a causal relationship between the use of inhaled antibiotics and $A$. fumigatus colonization. However most of our patients were using inhaled tobramycin at first isolation of Aspergillus.

There are few studies investigating the role of A. fumigatus on lung function in $\mathrm{CF}$, and these studies have not shown significant lung function decline in patients colonized with $A$. fumigatus..$^{21}$ Bargon et al. ${ }^{19}$ found no significant association between $A$. fumigatus colonization and lung function, in their adult CF patients. Although the decline of FEV1, FVC and FEF2575 in our cohort with Aspergillus colonization, these findings were not statistically significant. Other retrospective cohort analysis on 163 patients with Aspergillus colonization also did not find any differences regarding lung function decline during the study period. ${ }^{22}$ A causal relationship was not shown, but this study suggests that $A$. fumigatus may influence lung functions and risk of pulmonary exacerbations. However, in another crosssectional study with $7010 \mathrm{CF}$ patients from the European Epidemiologic Registry of Cystic
Fibrosis, Aspergillus colonization was associated with impaired lung functions. ${ }^{23}$ In Canada, a retrospective cohort study of pediatric nonABPA CF patients showed that two or more respiratory samples positive for A. fumigatus in any given year was associated with a significant reduction in FEV1 and a significant increase in pulmonary exacerbations requiring hospitalization compared with pediatric $\mathrm{CF}$ patients without $A$. fumigatus in respiratory samples. ${ }^{22}$

Noni et al. ${ }^{18}$ showed that $A$. fumigatus chronic colonization and lung function decline may have a causal relationship. Patients with $A$. fumigatus chronic colonization had significantly lower lung function in a seven-year prospective study period. Also baseline FEV1 was statistically different between groups before colonization and this may have led to the chronic colonization in these patients. Saunders et al. ${ }^{16}$ also reported that $A$. fumigatus colonization may be associated with worse lung functions.

Aspergillus sensitization and its association with poorer lung function in CF lung disease was investigated by a number of studies., Baxter et al. ${ }^{10}$ revealed that CF patients with Aspergillus sensitization showed a significantly higher FEV1 decline over two years compared to the group of patients without sensitization. We could not estimate the effect of Aspergillus sensitization on lung functions, because of the small number of patients in our cohort. Furthermore, Fillaux et al. ${ }^{3}$ reported that, ABPA, Aspergillus sensitization and persistent carriage have an impact on pulmonary functions in $\mathrm{CF}$ patients. Aspergillus bronchitis is also associated with lung function decline in patients with CF. 1,5 Aspergillus bronchitis led to decline in FEV1\%, $\mathrm{FVC} \%$ and $\mathrm{FEF} 25-75 \%$ in our cohort which is not statistically significant.

Kraemer et al. ${ }^{24}$ showed a significant negative effect of ABPA on FEV1 in a retrospective study with 122 mostly pediatric CF patients. Baxter et al. ${ }^{10}$ also reported, CF patients with ABPA had significant FEV1 decline over a two year study period compared to other patients without 
ABPA. Our findings also showed that the most prominent lung function decline was in patients with ABPA which was not statistically significant as shown in Figure 1.

Aspergillus colonization and infection are treated with antifungal agents such as azoles, however ABPA is usually treated with corticosteroids additionally with itraconazole or voriconazole. ${ }^{6,25}$ There is only one published prospective, randomized, controlled study investigating the effect of antifungal therapy on pulmonary outcomes in CF patients. ${ }^{26}$ Due to the retrospective nature of our study we have limited results about the effect of antifungal therapy on pulmonary outcome.

Our findings are limited by the retrospective nature of data collection and low numbers of the patients and the lack of longitudinal data for Aspergillus classification stages in the relevant subgroups. Also because this is a retrospective study of CF patients with Aspergillus growth, we could not compare the results with other CF patients as a control group. It can be assumed that patients can change within the different classification stages and that the classification status is probably not stable. We could not classify the patients depending on immunological results due to missing examinations of Aspergillus-specific IgG in these patients, we also defined sensitization, Aspergillus bronchitis, and colonization depending on the clinical criteria. Also due to a small number of patients having performed spirometry, evaluation of the lung function tests within years is difficult to estimate the prognosis of colonization, sensitization and ABPA in CF patients.

In conclusion, our study provides evidence for the significant effect of Aspergillus colonization on lung functions and emphasizes that chronic colonization should be considered pathogenic in CF patients. However, the most important question that requires addressing is the clinical significance of the fungi detected in $\mathrm{CF}$ and whether eradication needs consideration.
Prospective controlled studies with treatment arms are an important requirement for this field to progress.

\section{REFERENCES}

1. Chotirmall SH, Martin-Gomez MT. Aspergillus species in bronchiectasis: challenges in the cystic fibrosis and non-cystic fibrosis airways. Mycopathologia 2018; 183(Suppl 1): 45-59.

2. Chaudhary N, Datta K, Askin FB, Staab JF, Marr KA. Cystic fibrosis transmembrane conductance regulator regulates epithelial cell response to Aspergillus and resultant pulmonary inflammation. Am J Respir Crit Care Med 2012; 185: 301-310.

3. Fillaux J, Bremont F, Murris M, et al. Assessment of Aspergillus sensitization or persistent carriage as a factor in lung function impairment in cystic fibrosis patients. Scand J Infect Dis 2012; 44: 842-847.

4. Baxter CG, Moore CB, Jones AM, Webb AK, Denning DW. IgE-mediated immune responses and airway detection of Aspergillus and Candida in adult cystic fibrosis. Chest 2013; 143: 1351-1357.

5. Shoseyov D, Brownlee KG, Conway SP, Kerem E. Aspergillus bronchitis in cystic fibrosis. Chest 2006; 130: $222-226$

6. Schwarz C, Hartl D, Eickmeier O, et al. Progress in definition, prevention and treatment of fungal infections in cystic fibrosis. Mycopathologia 2018; 183: 21-32.

7. Singh A, Ralhan A, Schwarz C, Hartl D, Hector A. Fungal pathogens in CF airways: leave or treat? Mycopathologia 2018; 183: 119-137.

8. Cowley AC, Thornton DJ, Denning DW, Horsley A. Aspergillosis and the role of mucins in cystic fibrosis. Pediatr Pulmonol 2017; 52: 548-555.

9. Larone DH. Medically Important Fungi: A Guide to Identification.(5th ed).Washington: DC. ASM Press, 2011.

10. Baxter CG, Dunn G, Jones AM, et al. Novel immunologic classification of aspergillosis in adult cystic fibrosis. J Allergy Clin Immunol 2013; 132: 560-566.e10.

11. Jones AM, Horsley A, Denning DW. What is the importance of classifying Aspergillus disease in cystic fibrosis patients? Expert Rev Respir Med 2014; 8: 389-392.

12. Brandt C, Roehmel J, Rickerts V, Melichar V, Niemann N, Schwarz C. Aspergillus bronchitis in patients with cystic fibrosis. Mycopathologia 2018; 183: 61-69. 
13. Stevens DA, Moss RB, Kurup VP, et al; Participants in the Cystic Fibrosis Foundation Consensus Conference. Allergic bronchopulmonary aspergillosis in cystic fibrosis-state of the art: Cystic Fibrosis Foundation Consensus Conference. Clin Infect Dis 2003; 37(Suppl 3): S225-S264.

14. Ziesing S, Suerbaum S, Sedlacek L. Fungal epidemiology and diversity in cystic fibrosis patients over a 5-year period in a national reference center. Med Mycol 2016; 54: 781-786.

15. de Vrankrijker AM, van der Ent CK, van Berkhout FT, et al. Aspergillus fumigatus colonization in cystic fibrosis: implications for lung function? Clin Microbiol Infect 2011; 17: 1381-1386.

16. Saunders RV, Modha DE, Claydon A, Gaillard EA. Chronic Aspergillus fumigatus colonization of the pediatric cystic fibrosis airway is common and may be associated with a more rapid decline in lung function. Med Mycol 2016; 54: 537-543.

17. Maturu VN, Agarwal R. Prevalence of Aspergillus sensitization and allergic bronchopulmonary aspergillosis in cystic fibrosis: systematic review and meta-analysis. Clin Exp Allergy 2015; 45: 1765-1778.

18. Noni M, Katelari A, Dimopoulos G, Doudounakis SE, Tzoumaka-Bakoula C, Spoulou V. Aspergillus fumigatus chronic colonization and lung function decline in cystic fibrosis may have a two-way relationship. Eur J Clin Microbiol Infect Dis 2015; 34: 2235-2241.

19. Bargon J, Dauletbaev N, Kohler B, Wolf M, Posselt HG, Wagner TO. Prophylactic antibiotic therapy is associated with an increased prevalence of Aspergillus colonization in adult cystic fibrosis patients. Respir Med 1999; 93: 835-838.
20. Burns JL, Van Dalfsen JM, Shawar RM, et al. Effect of chronic intermittent administration of inhaled tobramycin on respiratory microbial flora in patients with cystic fibrosis. J Infect Dis 1999; 179: 1190-1196.

21. Speirs JJ, van der Ent CK, Beekman JM. Effects of Aspergillus fumigatus colonization on lung function in cystic fibrosis. Curr Opin Pulm Med 2012; 18: 632638.

22. Amin R, Dupuis A, Aaron SD, Ratjen F. The effect of chronic infection with Aspergillus fumigatus on lung function and hospitalization in patients with cystic fibrosis. Chest 2010; 137: 171-176.

23. Navarro J, Rainisio M, Harms HK, et al. Factors associated with poor pulmonary function: crosssectional analysis of data from the ERCF. European Epidemiologic Registry of Cystic Fibrosis. Eur Respir J 2001; 18: 298-305.

24. Kraemer R, Delosea N, Ballinari P, Gallati S, Crameri R. Effect of allergic bronchopulmonary aspergillosis on lung function in children with cystic fibrosis. Am J Respir Crit Care Med 2006; 174: 1211-1220.

25. King J, Brunel SF, Warris A. Aspergillus infections in cystic fibrosis. J Infect 2016; 72 (Suppl): S50-S55.

26. Aaron SD, Vandemheen KL, Freitag A, et al. Treatment of Aspergillus fumigatus in patients with cystic fibrosis: a randomized, placebo-controlled pilot study. PLoS One 2012; 7: e36077. 